(D) Thainá Lobato Calderoni ${ }^{1}$

(D) Yasmin Garcia Ribeiro ${ }^{1}$

(D) Mônica Feroni de Carvalho ${ }^{1}$

(D) Elizabeth Accioly ${ }^{2}$

(D) Naiara Sperandio ${ }^{1}$

(iD) Jane de Carlos Santana

Capelli ${ }^{1}$

1 Universidade Federal do Rio de Janeiro, Centro Multidisciplinar UFRJ-Macaé, Curso de Nutrição. Macaé, RJ, Brasil.

2 Universidade Federal do Rio de Janeiro, Instituto de Nutrição Josué de Castro, Departamento de Nutrição e Dietética. Rio de Janeiro, RJ, Brasil.

Correspondência

Thainá Lobato Calderoni tlc.thaina@gmail.com

\section{Construção e validação de um material educativo como estratégia de promoção da alimentação adequada e saudável na Atenção Básica}

\section{Construction and validation of an educational material as a strategy to promote proper and healthy food in Primary Care}

\section{Resumo}

Introdução: Os materiais educativos e didáticos distribuídos na Atenção Básica constituem importantes estratégias de promoção da saúde e de Educação Alimentar e Nutricional. Objetivo: Apresentar o processo de construção e validação de um material educativo sobre a alimentação de crianças menores de dois anos, dirigido aos profissionais de saúde. Método: Realizou-se estudo quantitativo com delineamento estruturado em duas etapas: construção e validação do material educativo. O processo de construção do material educativo se deu a partir da revisão da literatura e das atividades práticas desenvolvidas com mães e profissionais de saúde das unidades de saúde no município de Macaé-RJ. Na validação, o material educativo foi encaminhado por e-mail aos especialistas e, da mesma forma, após ajustes, enviado aos juízes. Calcularam-se as médias aritméticas, tendo como ponto de corte a nota $\geq 7$ de cada item para verificar a importância/adequação atribuída e os desvios-padrão, tendo como ponto de corte o valor <3 para estimar o grau de consenso entre os especialistas e juízes. Resultados: 0 miniguia apresenta três módulos temáticos: Aleitamento materno, Alimentação Complementar partes 1 e 2 . Na validação pelos especialistas, a média( $\pm D P)$ foi $8,5( \pm 1,2)$, sendo o material considerado importante/adequado e consensuado por todos; pelos juízes, a média( $\pm D P)$ foi 9,9( $\pm 0,3)$, sendo considerado importante/adequado e consensuado por todos. Conclusão: O miniguia foi considerado "importante/adequado" e consensuado pelos avaliadores.

Palavras-chave: Alimentação Infantil. Alimentação Complementar. Promoção da Saúde.

\footnotetext{
Abstract

Introduction: Educational and didactic materials available at the Primary Care units are important strategies to promote health and food and nutrition education. Objective: To present the construction and validation process of an educational material about feeding of children under the age of two years, designed to healthcare professionals. Method: a quantitative study was carried out with a design structured in two stages: construction and validation of the educational material. The construction process was based on a literature review and practical activities developed with mothers and health professionals in primary care units in the city of Macaé- RJ. For validation, the educational material was sent by e-mail to specialists and, likewise, after adjustments, to a panel of judges. Arithmetic means were calculated, having the score $\geq 7$ as the cutoff point of each item to verify the assigned importance/adequacy and standard
} 
DEMETRA

deviations. The cutoff value of $<3$ was set to estimate the degree of consensus among specialists and judges. Results: The miniguide has three thematic modules: Breastfeeding, Complementary Feeding - Parts 1 and 2. In the validation by specialists, the mean value $( \pm S D)$ was $8.5( \pm 1.2)$, and they all consensually considered the material important/appropriate; by judges, the mean value( \pm SD) was $9.9( \pm 0.3)$, being consensually considered important/appropriate. Conclusion: All evaluators in consensus agreed that the miniguide was "important/appropriate".

Keywords: Baby feeding. Complementary Feeding. Health Promotion., 


\section{INTRODUÇÃO}

A alimentação do lactente pode ser iniciada com a amamentação, ainda na sala do parto, de preferência até uma hora após o nascimento. ${ }^{1-3} \mathrm{O}$ aleitamento materno exclusivo (AME) até os seis meses de vida (e complementado até dois anos ou mais), seguido pela introdução da alimentação complementar (IAC), são recomendados pelo Ministério da Saúde (MS) por influenciarem de forma adequada e oportuna o estado nutricional do lactente, a fim de proporcionar o pleno crescimento e desenvolvimento, ${ }^{4,5}$ bem como a criação de futuros hábitos saudáveis para a vida adulta. ${ }^{6}$

No século XXI, por mais que a Organização Mundial da Saúde (OMS) e o MS mostrem a importância do AME e do incentivo à IAC no primeiro ano de vida, estudos brasileiros revelam que, em diversas regiões, ainda são oferecidos alimentos inapropriados às crianças menores de dois anos, principalmente nos primeiros seis meses de vida. ${ }^{7-10}$

No município de Macaé, Bouskelá et al., ${ }^{11}$ em estudo de série temporal avaliando a evolução das taxas de prevalência de AME aos quatro meses, entre 2001 e 2015, a partir de 56.454 registros do Departamento de Informática do Sistema Único de Saúde/ Ministério da Saúde/ Sistema de informação da Atenção Básica/ Sistema Único de Saúde (DATASUS/SIAB/SUS), revelaram que o AME apresentou aumento estatisticamente significativo de 47,2\% para 69,0\% entre 2001 e 2004. No período seguinte, as taxas de prevalência de AME na Atenção Básica à Saúde (ABS) de Macaé sofreram variações, sem significância estatística, observando-se o valor máximo de 76,8\%, no ano de 2013; e uma taxa anual média de 71,4\% nos últimos 10 anos.

Marinho et al., ${ }^{12}$ em um estudo de base secundária, com dados do Sisvan WEB, com 218 crianças de 6 a 24 meses, verificaram o elevado consumo de alimentos não saudáveis como, por exemplo, alimentos fontes de açúcar (mel, melado, açúcar simples e rapadura; 28,4\%), suco industrializado (50,5\%) e refrigerantes $(36,7 \%)$.

Diante desse cenário, inúmeras estratégias e ações de promoção da saúde e de educação alimentar e nutricional (EAN) voltadas ao grupo materno-infantil têm sido desenvolvidas no âmbito das políticas públicas brasileiras, de modo a promover e a incentivar a alimentação saudável, adequada e oportuna para esse grupo populacional. 4,6,13 Uma das estratégias de promoção da saúde e de EAN é a elaboração e a distribuição de materiais educativos e didáticos, que são importantes instrumentos de difusão de saberes e práticas desenvolvidas em diferentes territórios, ${ }^{14}$ e podem ser utilizados por profissionais de saúde na sua prática profissional, com o objetivo de orientar cuidadores sobre diferentes temas, como a alimentação nos dois primeiros anos de vida. ${ }^{15}$

No município de Macaé, as ações de EAN voltadas ao público materno-infantil e profissionais de saúde têm sido desenvolvidas pela Coordenadoria da Área Técnica de Alimentação e Nutrição (Catan), em parceria com o projeto de extensão universitária "Incentivo à Alimentação Complementar Adequada voltada aos Lactentes assistidos na Rede de Atenção à Saúde do Município de Macaé", conhecido como projeto IACOL, da Universidade Federal do Rio de Janeiro/Campus UFRJ-Macaé Professor Aloísio Teixeira, há cerca de oito anos. Uma das ações desenvolvidas é a realização de salas de espera na ABS, com distribuição de materiais educativos, tanto para mães usuárias das unidades básicas de saúde (UBS) como para profissionais que lá trabalham. Por ocasião dessas experiências em serviço, a equipe do IACOL foi instigada a elaborar material educativo mais denso, em linguagem de forma a atender profissionais e usuários da ABS, sobre o tema "Alimentação nos dois primeiros anos de vida com ênfase na alimentação complementar".

Nesse contexto, o presente estudo visa apresentar o processo de construção e validação de um material educativo sobre a alimentação de crianças menores de dois anos dirigido aos profissionais de saúde, com ênfase na alimentação complementar. 


\section{MÉTODOS}

Realizou-se estudo quantitativo com delineamento estruturado em duas etapas. A primeira compreendeu a construção de um material educativo, concebido inicialmente como cartilha, sobre alimentação saudável de crianças menores de dois anos, com ênfase na introdução da alimentação complementar. A segunda etapa foi a validação do material educativo em duas fases: (a) fase 1 - por especialistas de diferentes áreas da Nutrição; (b) fase 2 - por juízes, estudantes do ensino superior participantes de projeto de pesquisa e extensão na área da Saúde e Nutrição Materno-infantil (fluxograma 1). Foi concebida, inicialmente, uma terceira etapa de validação, envolvendo mães usuárias da ABS, mas que foi inviabilizada em razão da crise sanitária produzida pela pandemia da COVID-19.

O presente estudo faz parte do projeto de pesquisa matriz intitulado "Amamenta e Alimenta na Atenção Primária à Saúde do Município de Macaé-RJ", vinculado ao Núcleo de Ações e Estudos em Materno-Infantil (NAEMI), da UFRJ-Macaé, aprovado pelo Comitê de Ética em Pesquisa da Faculdade de Medicina de Campos dos Goytacazes/RJ, em 2014, sob o CAAE n³ 30378514.1.0000.5244.

Fluxograma 1. Processo de construção e validação do material educativo.

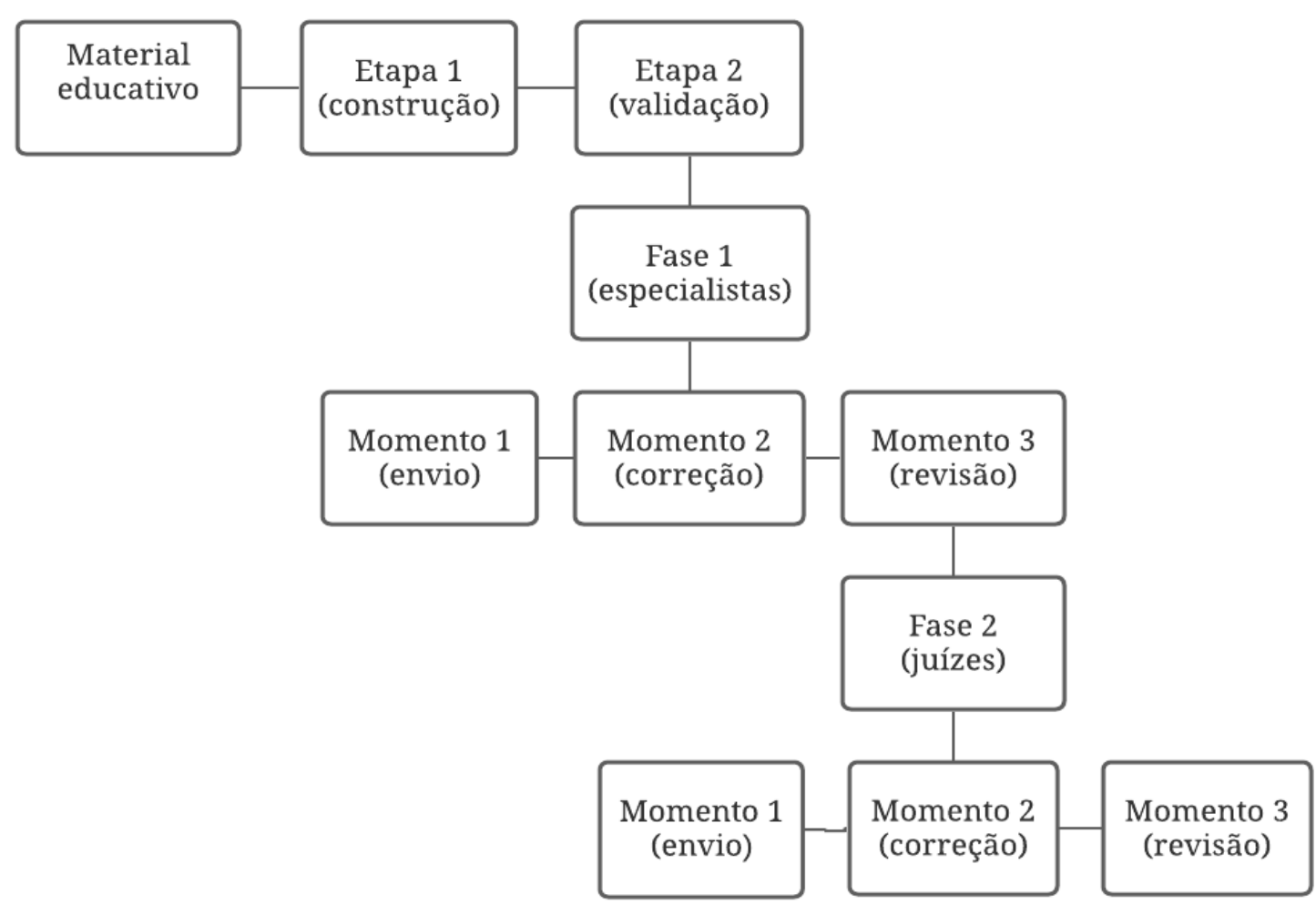

\section{ETAPA 1. Processo de construção do material educativo}

Realizou-se levantamento bibliográfico a partir das bases de dados da Biblioteca Virtual em Saúde (BVS): Medline da Virtual Health Library (VHL), Literatura Latino-Americana e do Caribe em Ciências da Saúde (Lilacs), Scientific Eletronic Library Online Brasil) (Scielo Brasil); e PubMed, além de informes 
técnicos nas webpages do MS, da Sociedade Brasileira de Pediatria (SBP) e da Organização Panamericana da Saúde (OPAS)/OMS. As buscas nas bases de dados foram conduzidas a partir de junho de 2019, utilizando um conjunto de descritores, em língua portuguesa, do site Descritores em Ciências da Saúde (DeCS) e no Medical Subject Headings (MESH): Alimentação, Alimentação Complementar, Alimentação Infantil, Criança, Lactente e Práticas Alimentares. Foram selecionados os artigos que abordavam o processo de construção de materiais educativos, estudos epidemiológicos voltados à alimentação de crianças menores de dois anos, bem como guias alimentares e textos contendo definições, conceitos, leis e decretos sobre o tema.

O conteúdo do material educativo foi estruturado a partir de módulos temáticos (Aleitamento materno, Alimentação complementar partes 1 e 2) definidos após a revisão da literatura e nas experiências obtidas pela equipe do projeto IACOL em salas de espera e oficinas com gestantes, puérperas, nutrizes, cuidadores de lactentes, graduandos de diferentes áreas da saúde, comunidade em geral e profissionais de saúde, realizadas nas UBS e de ESF de Macaé. O material foi construído segundo as recomendações para concepção e eficácia de materiais educativos, de acordo com os seguintes atributos: conteúdo, linguagem, ilustrações, layout, motivação e cultura. ${ }^{16,17}$

A linguagem, o layout e a ilustração da cartilha foram concebidos para serem de fácil compreensão e interesse do público-alvo. As imagens foram cedidas do acervo do projeto IACOL bem como do acervo pessoal da pesquisadora do estudo. Algumas imagens foram cedidas por mães e cuidadores para a pesquisadora, sendo devidamente autorizadas por meio da assinatura do termo de cessão de direitos de uso de imagem encaminhado previamente por e-mail.

Inicialmente, para a criação da cartilha, utilizou-se o programa Microsoft $®$ PowerPoint for Windows 2020. Após a sua construção, a cartilha foi submetida a um profissional de ilustração e design para o desenvolvimento de layout, formatação, diagramação e ilustrações.

\section{ETAPA 2. Processo de validação do material educativo}

\section{Fase 1}

A validação do conteúdo da cartilha foi realizada utilizando-se a técnica conferência de consenso adaptada de Ferraro et al., ${ }^{18}$ Souza et al., ${ }^{19}$ Murphy et al., ${ }^{20}$ proposta por Ribeiro \& Spadella, ${ }^{15}$ visando "alcançar uma decisão em comum acordo entre duas ou mais partes, conciliando discussão aberta e o anonimato dos especialistas, em uma logística viável". A fase 1 foi estruturada em 3 momentos.

\section{Momento 1}

A versão preliminar da cartilha foi enviada por e-mail a uma equipe de sete profissionais, considerados especialistas de diferentes áreas da nutrição (quadro 1) para avaliação, em amostragem por conveniência, constituindo quatro docentes doutores em diferentes campos de atuação da Nutrição e três nutricionistas atuantes na ABS do município de Macaé. 
Quadro 1. Caracterização dos especialistas convidados para o estudo. Maio, 2020.

\begin{tabular}{|c|c|c|}
\hline Especialistas & Formação & Área de atuação/ tempo \\
\hline ESP 1 & $\begin{array}{l}\text { Nutricionista graduada pela Universidade } \\
\text { Federal Fluminense. Mestre em Atenção Primária } \\
\text { à Saúde pela Universidade Federal do Rio de } \\
\text { Janeiro (UFRJ). }\end{array}$ & $\begin{array}{l}\text { Saúde Coletiva (17 anos)/ Secretaria de } \\
\text { Atenção Básica/ Secretaria Municipal } \\
\text { de Saúde (SEMUSA). }\end{array}$ \\
\hline ESP 2 & $\begin{array}{l}\text { Nutricionista graduada pela Universidade do } \\
\text { Estado do Rio de Janeiro (UERJ). Doutora em } \\
\text { Ciências (UERJ). }\end{array}$ & Materno-infantil (10 anos). \\
\hline ESP 3 & Nutricionista. & $\begin{array}{l}\text { Educação Alimentar e Nutricional e } \\
\text { Nutrição Clínica (19 anos). }\end{array}$ \\
\hline ESP 4 & $\begin{array}{l}\text { Nutricionista graduada pela UFRJ. Doutora em } \\
\text { Ciências Nutricionais pela UFRJ. Mestre em } \\
\text { Nutrição Humana (UFRJ). }\end{array}$ & Saúde Coletiva (12 anos). \\
\hline ESP 5 & $\begin{array}{l}\text { Nutricionista pela UFRJ. Doutora em Alimentação, } \\
\text { Nutrição e Saúde pela UERJ. Mestre em Nutrição } \\
\text { Humana (UFRJ). }\end{array}$ & 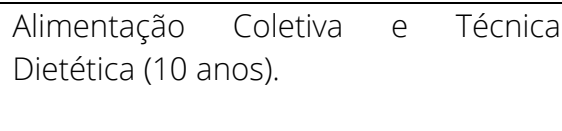 \\
\hline ESP 6 & Nutricionista graduada pela UFRJ. & $\begin{array}{l}\text { Nutrição Clínica e Nutrição Materno- } \\
\text { Infantil (20 anos). }\end{array}$ \\
\hline ESP 7 & $\begin{array}{l}\text { Nutricionista. Doutora em Educação em Ciências } \\
\text { e Saúde, Núcleo de Tecnologia Educacional para } \\
\text { Saúde pela Universidade Federal do Rio de } \\
\text { Janeiro (UFRJ). Mestre em Alimentação, Nutrição } \\
\text { e Saúde (UERJ). }\end{array}$ & $\begin{array}{l}\text { Educação Alimentar e Nutricional e } \\
\text { Saúde Coletiva ( } 7 \text { anos). }\end{array}$ \\
\hline
\end{tabular}

Fonte: Elaborado pelas próprias autoras.

Foram definidos os seguintes critérios de inclusão para definição do grupo de especialistas: atuar na área de Nutrição, com expertise em assistência nutricional materno-infantil, saúde coletiva ou técnica dietética e tempo mínimo de experiência profissional de sete anos.

Junto ao envio da cartilha, foram adicionados o termo de consentimento livre e esclarecido (TCLE) e o instrumento de avaliação do material educativo. Foi solicitado que os especialistas lessem cuidadosamente o material e encaminhassem contribuições e sugestões, e retornassem por e-mail em um prazo de até 15 dias.

O instrumento de avaliação do material educativo foi elaborado com adaptações a partir do instrumento de Sousa \& Turrini ${ }^{16}$ contendo as seguintes categorias de avaliação, subdivididas em vários itens de análise: conteúdo, linguagem, ilustrações, layout, motivação e cultura, com notas variando de zero a dez para cada item. O instrumento continha um campo denominado observações, para os especialistas registrarem suas sugestões, observações e contribuições.

\section{Momento 2}

Realizou-se a correção da cartilha, a partir das contribuições encaminhadas pelos especialistas que, por sugestão de alguns desses avaliadores, passou a ser denominada "miniguia" sobre alimentação saudável de crianças menores de dois anos, com ênfase na introdução da alimentação complementar, por ser considerada uma boa extração sintetizada do Guia Alimentar para Crianças Brasileiras Menores de Dois Anos. $^{2}$ 
As notas atribuídas a cada item foram transferidas para a planilha do programa Excel (Microsoft, Corporation, USA). Foram calculadas as médias aritméticas de cada item para verificar a importância/adequação atribuída, bem como os desvios-padrão para estimar o grau de consenso entre os especialistas. ${ }^{18}$

Foram adotados como pontos de corte: média $\geq 7$ como item importante/adequado; média < 7 como item pouco importante/adequado; desvio padrão <3 como resultado consensual e desvio padrão $\geq 3$ com resultado não consensual, adaptados de Souza e colaboradores. ${ }^{19}$

\section{Momento 3}

Os pontos concordantes entre as observações feitas pelos especialistas, bem como as sugestões apresentadas, nortearam as alterações feitas no miniguia realizando-se, nesse momento, a revisão final do material educativo para envio aos juízes.

\section{Fase 2}

O miniguia revisado, o instrumento e o TCLE foram enviados por e-mail para leitura, contribuições e sugestões no material para a equipe de juízes $(n=10)$, compostos por graduandos dos cursos de Nutrição e Medicina, da UFRJ-Macaé, integrantes de um projeto de extensão e de pesquisa na área de saúde MaternoInfantil, por atuarem nesse campo do conhecimento (momento 1). Cabe ressaltar que os graduandos do estudo foram escolhidos aletoriamente e assinaram o TCLE como participantes desta etapa da pesquisa.

Analisaram-se as contribuições dadas pelos avaliadores e, posteriormente, as notas atribuídas em cada item dos formulários foram digitadas em uma planilha do programa Excel (Microsoft, Corporation, USA). Calcularam-se a média aritmética e o desvio-padrão de cada item. ${ }^{18}$ Os mesmos pontos de corte, já descritos anteriormente, foram adotados (momento 2).

O miniguia passou por uma revisão final pela pesquisadora principal (momento 3), na qual foram consideradas pelo menos três falas recorrentes de diferentes especialistas $(43,0 \%)$ e quatro falas de juízes $(40,0 \%)$ para a revisão do material educativo.

Neste estudo, para a elaboração dos resultados, algumas das principais falas foram apresentadas no corpo do texto, mantendo-se preservada a identidade dos participantes por meio de codinomes.

\section{RESULTADOS}

O processo de construção e validação do miniguia ocorreu no período maio de 2018 a dezembro 2020, a partir da revisão da literatura, das experiências e vivências acumuladas nas ações desenvolvidas pelo projeto IACOL na Rede de Atenção à Saúde (RAS) do município de Macaé. O miniguia intitula-se "Alimentando o seu bebê - Miniguia com orientações básicas para escolhas alimentares adequadas" e apresenta três módulos temáticos (Aleitamento Materno; Alimentação Complementar - partes 1 e 2) e seus títulos, totalizando 47 páginas, com ilustrações.

Em relação à validação pelos especialistas, verificou-se a média( \pm DP) de $8,5( \pm 1,2)$ dos itens avaliados quanto a conteúdo, linguagem, ilustrações, layout, motivação e cultura, considerando-se o material educativo "importante/adequado" e com o consenso dos especialistas (tabela 1). 
Tabela 1. Médias e desvios padrão dos itens de avaliação do miniguia conferido pelos especialistas, segundo as etapas de validação. Macaé-RJ, maio a julho, 2020 (n=7).

\begin{tabular}{|c|c|}
\hline Itens de Avaliação & Média $\pm D P$ \\
\hline \multicolumn{2}{|l|}{ Conteúdo } \\
\hline $\begin{array}{l}\text { O conteúdo abordado é relevante para a promoção da alimentação saudável para crianças } \\
\text { menores de } 2 \text { anos. }\end{array}$ & $10,0 \pm 0,0$ \\
\hline $\begin{array}{l}\text { O conteúdo está apropriado ao público-alvo (mãe e cuidadores de crianças menores de } 2 \\
\text { anos). }\end{array}$ & $9,1 \pm 0,8$ \\
\hline O conteúdo é suficiente para atender às necessidades do público-alvo. & $9,6 \pm 0,7$ \\
\hline O conteúdo é de fácil aplicabilidade no cotidiano do público-alvo. & $9,6 \pm 0,7$ \\
\hline \multicolumn{2}{|l|}{ Linguagem } \\
\hline O estilo da redação é compatível com o público-alvo. & $8,7 \pm 1,4$ \\
\hline A escrita utilizada é atrativa. & $8,3 \pm 1,9$ \\
\hline A linguagem é clara e objetiva. & $8,7 \pm 2,1$ \\
\hline \multicolumn{2}{|l|}{ Ilustrações } \\
\hline As ilustrações são pertinentes com o conteúdo do material e elucidam esse conteúdo. & $8,9 \pm 2,1$ \\
\hline As ilustrações são claras e transmitem facilidade de compreensão. & $9,6 \pm 1,0$ \\
\hline $\begin{array}{l}\text { A quantidade de ilustrações está adequada para o conteúdo d material de apoio. } \\
\text { Layout }\end{array}$ & $9,9 \pm 0,3$ \\
\hline O tipo de letra utilizado facilita a leitura. & $9,9 \pm 0,3$ \\
\hline As cores aplicadas ao texto são pertinentes e facilitadoras para a leitura. & $9,7 \pm 0,7$ \\
\hline A composição visual está atrativa e bem organizada. & $9,7 \pm 0,7$ \\
\hline O formato (tamanho) do material de apoio e o número de páginas estão adequadas. & $10,0 \pm 0,0$ \\
\hline A disposição do texto está adequada. & $9,7 \pm 0,7$ \\
\hline O tamanho das letras dos títulos, subtítulos e textos estão adequados. & $9,9 \pm 0,6$ \\
\hline \multicolumn{2}{|l|}{ Motivação } \\
\hline O conteúdo está motivador e incentivará a prosseguir com a leitura. & $9,4 \pm 1,0$ \\
\hline O conteúdo despertará interesse nos leitores. & $9,3 \pm 1,4$ \\
\hline O conteúdo atende as dúvidas, esclarece e educa o público-alvo. & $9,0 \pm 2,4$ \\
\hline \multicolumn{2}{|l|}{ Cultura } \\
\hline $\begin{array}{l}\text { O texto está compatível com o público-alvo, atendendo aos diferentes perfis de } \\
\text { conhecimento. }\end{array}$ & $8,3 \pm 2,2$ \\
\hline Média geral & $8,5 \pm 1,2$ \\
\hline
\end{tabular}

Fonte: Elaborado pelas autoras.

Na etapa de validação realizada pelos especialistas, foram sinalizados os aspectos relacionados ao conteúdo, à linguagem, às ilustrações, ao layout, à cultura e à motivação/elogio. Quanto ao conteúdo, verificou-se a necessidade de adicionar, complementar e rescrever alguns pontos como, por exemplo,

Deve-se atentar a lista de ingredientes no rótulo dos alimentos. Vão dar alguma orientação para olhar o rótulo? (Esp. 3)

Por exemplo, combinar com familiares e amigos quem pode limpar a casa, fazer a comida, lavar e passar a roupa, de modo que a mãe possa se concentrar na amamentação e não se sobrecarregar. É comum nas periferias as mulheres, por exemplo contarem com o apoio de pessoas que não são da família [...]. (Esp. 7)

Sugestão do bolo de banana - retirar o açúcar (conferir recomendação nas preparações para crianças menores de 2 anos) e mudar pela maçã ralada. Se a banana estiver bem madura, não há necessidade da adição do açúcar para o paladar infantil (zero viciado em açúcar). (Esp. 5) 
No que tange à linguagem, considerou-se a necessidade de melhorar o diálogo, diminuir e substituir alguns termos apresentados.

A palavra lactente poderia ser substituída pela palavra bebê. Isso padronizaria a linguagem do material e poderia ser mais bem compreendida pelo público geral. (Esp. 2)

Tenho a impressão que alguns verbos e suas conjugações trazem uma ideia de ordem: dever, por exemplo. Palavras como saciedade, safra, composição nutricional, monotonia... talvez não comuniquem. (Esp. 7)

Já em relação às ilustrações, houve a necessidade de incluir imagens que caracterizassem melhor o perfil da nutriz e do lactente atendidos pelo profissional de saúde, na ABS de Macaé, no que tange à etnia/raça/cor.

[...] Duas fotos de mulheres amamentando: duas mulheres de cabelo liso, brancas; ao pensar nas pessoas que vão receber esse material é muito importante para a comunicação que elas se sintam incluídas nesse material [...]. Contemplar crianças negras, mães negras, familiares negros, uma família. (Esp 5)

Houve também sugestões para melhorar o layout, a fim de destacar e torná-lo mais lúdico durante a leitura. Desta forma, incluíram-se imagens a partir destas considerações.

No item 2.2. Poderia colocar na cartilha alguns destaques para algumas informações com cor diferente e com a palavra DICA, ATENÇÃO. No item 4.6 acho que poderia colocar um espaçamento entre as linhas no texto para não ficar muito grudado. Não precisa ser de 1,5, mas 1,15 já ajuda na visualização do texto. (Esp. 3)

[...] as imagens dos pratos foram mais que perfeitas. Sugiro que o gráfico de pizza (p.12) também seja transformado em prato com comida [...]. Poderia ser interessante que as cores das chamadas fossem iguais quando os temas forem dentro de uma mesma dimensão. Por exemplo: azul para aleitamento, (...). (Esp. 7).

Por fim, houve a sugestão de incluir assuntos em relação à cultura, como:

Talvez vocês pudessem sugerir que os produtores locais fossem privilegiados em relação aos grandes mercados por conta da questão agroecológica e que aquele alimento percorreu menos estrada, está mais fresco, questão da economia local. Talvez sugerir que deem preferência aos consumidores, feirinha do bairro. (Esp. 5)

Cabe ressaltar que muitos elogios/motivação foram feitos pelos especialistas, podendo ser destacados,

Material excelente! Bonito, atrativo, interessante, temas abrangentes e aprofundados na temática escolhida para a pesquisa. (Esp. 1)

Pensando que esse material vai ser muito útil, ela está muito objetiva, está como se fosse uma extração do guia (um extrato mesmo), acho desde esse momento, onde esse material pode inspirar nutricionistas e também pode acabar dentro das mãos das mães que ele possa se comunicar de forma ampla, inclusiva com esse espelhamento [...] Parabéns, trabalho lindo. Está muito bonito, muito necessário, muito objetivo. [...] vai ser de muita utilidade. (Esp. 5) 
Na validação realizada pelos juízes, observou-se a média geral $( \pm D P)$ de $9,9( \pm 0,3)$ dos itens avaliados, sendo considerada "importante/adequado" com o consenso dos juízes (tabela 2).

Tabela 2. Médias e desvios padrão dos itens de avaliação do miniguia conferido pelos juízes, segundo as etapas de validação. Macaé-RJ, setembro, 2020 (n=10).

\begin{tabular}{|c|c|}
\hline Itens de Avaliação & Média $\pm \mathrm{DP}$ \\
\hline \multicolumn{2}{|l|}{ Conteúdo } \\
\hline $\begin{array}{l}\text { O conteúdo abordado é relevante para a promoção da alimentação saudável para } \\
\text { crianças menores de } 2 \text { anos. }\end{array}$ & $10,0 \pm 0,0$ \\
\hline $\begin{array}{l}\text { O conteúdo está apropriado ao público-alvo (mãe e cuidadores de crianças menores de } \\
2 \text { anos). }\end{array}$ & $10,0 \pm 0,0$ \\
\hline O conteúdo é suficiente para atender às necessidades do público-alvo. & $9,5 \pm 0,5$ \\
\hline Linguagem & $9,5 \pm 0,4$ \\
\hline O estilo da redação é compatível com o público-alvo. & $10,0 \pm 0,0$ \\
\hline A escrita utilizada é atrativa. & $10,0 \pm 0,4$ \\
\hline A linguagem é clara e objetiva. & $10,0 \pm 0,6$ \\
\hline llustrações & $10,0 \pm 0,0$ \\
\hline As ilustrações são pertinentes com o conteúdo do material e elucidam esse conteúdo. & $10,0 \pm 0,0$ \\
\hline As ilustrações são claras e transmitem facilidade de compreensão. & $10,0 \pm 0,0$ \\
\hline $\begin{array}{l}\text { A quantidade de ilustrações está adequada para o conteúdo do material de apoio. } \\
\text { Layout }\end{array}$ & $10,0 \pm 0,3$ \\
\hline O tipo de letra utilizado facilita a leitura. & $9,5 \pm 0,4$ \\
\hline As cores aplicadas ao texto são pertinentes e facilitadoras para a leitura. & $10,0 \pm 0,6$ \\
\hline A composição visual está atrativa e bem organizada. & $10,0 \pm 0,0$ \\
\hline O formato (tamanho) do material de apoio e o número de páginas estão adequadas & $9,5 \pm 0,3$ \\
\hline A disposição do texto está adequada. & $10,0 \pm 0,3$ \\
\hline $\begin{array}{l}\text { O tamanho das letras dos títulos, subtítulos e textos estão adequados. } \\
\text { Motivação }\end{array}$ & $10,0 \pm 0,6$ \\
\hline O conteúdo está motivador e incentivará a prosseguir com a leitura. & $9,5 \pm 0,3$ \\
\hline O conteúdo despertará interesse nos leitores. & $9,5 \pm 0,4$ \\
\hline $\begin{array}{l}\text { O conteúdo atende as dúvidas, esclarece e educa o público-alvo. } \\
\text { Cultura }\end{array}$ & $9,0 \pm 0,6$ \\
\hline $\begin{array}{l}\text { O texto está compatível com o público-alvo, atendendo aos diferentes perfis de } \\
\text { conhecimento. }\end{array}$ & $9,0 \pm 0,6$ \\
\hline Média geral & $9,9 \pm 0,3$ \\
\hline
\end{tabular}

Fonte: Elaborado pelas autoras.

Na validação do miniguia realizada pelos juízes, já com as alterações feitas a partir das orientações dos especialistas, foi apontada a necessidade de melhorar a linguagem e o layout. Em relação à linguagem, os juízes destacaram a existência de repetições de termos utilizados no material, como por exemplo:

Ressalto a repetição de palavras, como "mãe", "bebê", que pode tornar a leitura por vezes cansativa. (Juiz 9)

Quanto ao layout, houve sugestões como:

Aumentar um pouquinho a faixa laranja na capa para encaixar melhor todos os nomes das autoras; na segunda página em rosa [...] percebi haver muito espaço entre os textos; tenho o costume de colocar sempre um recuo nas primeiras linhas de cada parágrafo [...] senti 
falta disso, mas acredito não ser obrigatório, foi apenas uma observação que fiz pelo costume que tenho, fui até conferir no Guia de 2019 e lá está como o seu. (Juiz 6)

O miniguia também recebeu inúmeros elogios dos juízes, o que indicou a qualidade e a contribuição do mesmo para os profissionais de saúde que atendem os usuários da ABS:

Parabéns pelo trabalho. Material excelente, de fácil compreensão, lúdico e layout muito bem feito [...]. (Juiz 1)

Os pontos que achei mais evidencia são a relevância do conteúdo para a sociedade e a linguagem que é feita de forma clara e acessível a todo o público. (Juiz 10)

Após a revisão do material educativo, em cada etapa de validação, ele passou por uma revisão final pela pesquisadora e foi encaminhado para diagramação final. Em paralelo, elementos do miniguia, tais como: título, autoras, cidade, local da publicação, ano da publicação, número de páginas, tamanho, palavras-chave, biblioteca e bibliotecária, foram enviados à biblioteca do UFRJ-Macaé para elaboração da ficha catalográfica.

A versão final do miniguia foi encaminhada à editora do Observatório da Cidade de Macaé para avaliação, que o aprovou para disponibilizar na versão em e-book no formato pdf, ${ }^{21}$ pelo site do próprio Observatório.

\section{DISCUSSÃO}

O miniguia foi construído e validado no período de 31 meses. Na etapa de validação, ele foi avaliado por sete especialistas e 10 juízes que, segundo os resultados obtidos, consideraram o material educativo "importante/adequado", sendo consensuado por todos. As principais considerações feitas pelos especialistas foram relacionadas ao conteúdo, à linguagem, às ilustrações, ao layout e à cultura, podendo-se destacar: adicionar informações sobre rotulagem de alimentos e compartilhamento de tarefas domésticas; tornar o material mais acessível à população por meio do diálogo; adicionar imagens de mulheres e crianças negras e pardas; utilizar cores e destaques para estimular a leitura do material pelo usuário; incluir o uso de temperos e alimentos comprados em feiras regionais nas preparações. As principais considerações feitas pelos juízes foram relacionadas à linguagem e ao layout como, por exemplo, revisão ortográfica, repetição de algumas palavras e sugestões para o layout.

O material educativo é um dos métodos utilizados no processo de ensino-aprendizagem em saúde 22 e pode ser utilizado em ações de promoção da saúde voltadas aos diferentes grupos populacionais. Isto porque se constitui em um método palpável, tanto para o educador, como para o educando (público-alvo), por apresentar seu conteúdo elaborado de forma simples, ter informações que podem ser visualizadas facilmente, de modo a contribuir na melhoria da assimilação do tema proposto pelo público-alvo, após uma ação educativa. ${ }^{23}$

Nessa perspectiva, o conteúdo do material educativo deve ser definido a partir da sua proposta inicial (Qual o objetivo do infográfico? O que se deseja transmitir ao leitor?); do conteúdo de informações (Quais informações são necessárias para realizar o objetivo?); dos recursos (humanos e materiais); do meio de produção (Como será desenvolvido? Ilustração? Fotografia? Em quais softwares?); dos usuários (Para quem é destinado o infográfico?); e das circunstâncias do uso (Em qual mídia será vista e como será vista a publicação?). ${ }^{24}$ 
Neste estudo, o processo de construção e validação do material educativo foi pensado inicialmente para atender às demandas dos profissionais de saúde e as mães usuárias da ABS. Porém, no período da validação, foi declarada pela OMS a pandemia da COVID-19, o que impossibilitou a validação do material pelas usuárias das unidades, em cuja etapa se planejou realizar uma roda de conversa com gestantes, puérperas e nutrizes na unidade de saúde.

O projeto de extensão universitária IACOL tem como objetivo promover alimentação adequada e saudável nos primeiros dois anos de vida, com ênfase na alimentação complementar, tendo como públicoalvo das ações: gestantes, puérperas, nutrizes, cuidadores de lactentes, graduandos de diferentes áreas da saúde, comunidade em geral e profissionais de saúde. A partir das experiências acumuladas ao longo de cinco anos pela equipe do projeto nas salas de espera, nas oficinas ministradas nas diferentes unidades de saúde, hospitais (público e filantrópico), oficinas realizadas em eventos da UFRJ-Macaé, ${ }^{25-27}$ surgiu a necessidade de desenvolver um novo material que aprofundasse os conteúdos sobre alimentação complementar.

Cabe ressaltar que os profissionais de saúde participantes das ações, principalmente aqueles inseridos nas ESF de Macaé, em especial os enfermeiros, verbalizaram positivamente sobre o material educativo (folder) distribuído nas ações, porém sugeriam que ele poderia ser mais completo, contendo, por exemplo, receitas e orientações sobre a introdução de alimentos a partir dos seis meses. Isto porque as unidades da ESF não contam com nutricionistas em suas equipes, ${ }^{28}$ e sua elaboração serviria de apoio aos atendimentos realizados no cotidiano da prática profissional.

O miniguia, portanto, foi desenvolvido na perspectiva de aprofundar conteúdos relevantes e atualizados sobre a alimentação de crianças menores de dois anos, com ênfase na alimentação complementar e voltado para os profissionais de saúde de Macaé.

Uma abordagem indicada pelos especialistas para ser inserido no miniguia foi a rotulagem nutricional, ${ }^{21}$ uma vez que ainda há desconhecimento de parte da população e dos profissionais de saúde sobre as informações nutricionais presentes nos rótulos ou embalagens dos alimentos processados e ultraprocessados.

A rotulagem de alimentos é uma estratégia utilizada pela indústria alimentícia para informar ao consumidor sobre a composição nutricional e outras informações do alimento que está sendo comercializado, sendo regulamentada pela Agência Nacional de Vigilância Sanitária (Anvisa), na Resolução RDC n. 259, de 20 de setembro de 2002. ${ }^{29}$ Somada ao aumento da prevalência do sobrepeso, da obesidade e de carências nutricionais de micronutrientes, a rotulagem nutricional se tornou um tema de grande relevância na Política Nacional de Alimentação e Nutrição - PNAN, ${ }^{30}$ em sua segunda diretriz, Promoção da Alimentação Adequada e Saudável - PAAS. ${ }^{30}$

O tema da rotulagem nutricional, portanto, foi considerado relevante para ser inserido no material educativo, de modo a auxiliar o público-alvo no entendimento das informações nutricionais contidas nos rótulos de alimentos que, na maioria das vezes, não são compreendidas ou não são facilmente compreensíveis pelo consumidor (por exemplo, tamanho de letra pequena, cor da letra, cor da embalagem ou rótulo no qual a informação está descrita). ${ }^{31,32}$

O compartilhamento de tarefas domésticas como, por exemplo, rede de apoio à amamentação e preparo de refeições, foi outro assunto abordado pelos especialistas para ser introduzido no conteúdo. Essas sugestões foram acatadas pela pesquisadora que incorporou o tema no miniguia. ${ }^{21}$ 
O Guia Alimentar para Crianças Brasileiras Menores de Dois Anos, publicação do MS, aponta a rede de apoio como sendo o conjunto de pessoas e instituições que compõem os elos de relacionamentos do indivíduo e que contribuem para o seu apoio e sua proteção física, psicológica e emocional. ${ }^{2}$ Tanto amamentar como alimentar uma criança envolve o apoio de familiares e de profissionais de saúde, a fim de orientar e superar as dificuldades encontradas pelas mulheres e suas famílias. Com isso, a formação de uma rede de apoio para essa mãe pode se tornar um fator determinante durante a amamentação, a introdução de novos alimentos e a criação de hábitos saudáveis envolvendo a criança. ${ }^{33}$

O ato de cozinhar também foi mencionado no Guia Alimentar para Crianças Brasileiras Menores de Dois Anos, que está alinhado ao Guia Alimentar para a População Brasileira, ${ }^{33}$ apresenta uma abordagem pioneira ao buscar apoiar e dialogar sobre alimentação nos dois primeiros anos com as pessoas que cuidam da criança, como a mãe, o pai, as parceiras e/ou os parceiros da mães e do pai, dos avós, dos avôs, dos homens e das mulheres, com ou sem relação de parentesco, que morem ou não na mesma casa da criança, ${ }^{2}$ bem como apoiar os membros da comunidade e profissionais de saúde, educação e assistência social envolvidos no cuidado das crianças em seus locais de trabalho (unidades básicas de saúde, hospitais, creches etc.) e em todos os ambientes que promovam a alimentação adequada e saudável das crianças. ${ }^{2}$

Os especialistas sugeriram a retirada do ingrediente açúcar na receita do bolo de banana, indicada para crianças maiores de um ano, sendo acatada essa orientação. De acordo com o Guia, recomenda-se que não sejam ofertados alimentos e preparações adoçadas com qualquer tipo de açúcar, edulcorante calórico ou mel, pois pode ser um fator de risco para o ganho de peso na infância - tendo como consequência, o aparecimento de obesidade e de doenças crônicas associadas -, e a formação de placa bacteriana e cárie dentária. ${ }^{2}$

Desta forma, as duas receitas do miniguia, bolo de banana e mousse de abacate com banana e cacau, não tiveram a indicação de adição de açúcar, edulcorante e mel. Seguindo a recomendação de uma especialista, foram dadas como opção para adoçar as preparações, as frutas maçã ralada ou banana bem madura. ${ }^{21}$

Nos materiais educativos, é priorizada a linguagem dialógica com tom coloquial, de forma clara e simples visando estimular o pensamento e a reflexão por meio de perguntas. ${ }^{24}$ Neste sentido, os apontamentos apresentados pelos especialistas e juízes para melhorar a linguagem foram acatados para facilitar o diálogo entre o profissional de saúde e os usuários da unidade de saúde. A elaboração de um material contendo uma linguagem acessível, mesmo para profissionais de saúde, facilita o entendimento do conteúdo, promove autonomia ${ }^{33}$ e permite que eles possam transmitir as informações de forma mais simples aos usuários.

Para finalizar, foi possível perceber o interesse e a motivação dos especialistas e juízes pelo miniguia, uma vez que todos teceram elogios ao material, que alcançou a pontuação média acima de 8,5, indicando ser o miniguia importante/adequado, com desvio padrão abaixo do ponto de corte estabelecido (<3), o que indica consenso por todos os avaliadores.

A principal limitação do estudo foi a impossibilidade de validação do material pelas mães usuárias das unidades de saúde de Macaé (gestantes, puérperas e nutrizes), em razão do isolamento e distanciamento social devido à pandemia da COVID-19, e com o Decreto municipal de Macaé nº 30/2020,34 que determinou a paralisação das atividades acadêmicas. No entanto, já está sendo produzido um material educativo simplificado e ilustrado destinado a este público, como desdobramento do miniguia, para ser validado após o fim da pandemia no Brasil. Entende-se que esse material também será de grande importância para apoiar as ações educativas desenvolvidas tanto pelo projeto de extensão como profissionais de saúde da ABS. 


\section{CONCLUSÃO}

O material educativo foi considerado "importante/adequado", sendo consensuado por todos os avaliadores. O conteúdo, a linguagem, as ilustrações, o layout e a cultura foram os itens indicados para serem alterados no miniguia. A rotulagem nutricional, o compartilhamento de tarefas domésticas, o uso de temperos e os alimentos comprados em feiras regionais nas preparações foram assuntos indicados pelos avaliadores para serem introduzidos no miniguia.

Desta forma, o miniguia é um material educativo que contém informações atualizadas e aprofundadas sobre alimentação da criança menores de dois anos com ênfase na alimentação complementar, que apoiará e instrumentalizará os profissionais de saúde em sua rotina de trabalho, sendo acessível por meio de equipamentos eletrônicos através de mídia digital.

\section{REFERÊNCIAS}

1. World Health Organization/United Nations Children's Fund. Innocenti Declaration on the protection, promotion, and support of breastfeeding. Meeting "Breast-feeding in the 1990s: A global initiative". Florence (Italy): WHO/ UNICEF; 1990.

2. Brasil. Ministério da Saúde. Secretaria de Atenção Primária à Saúde. Departamento de Promoção da Saúde. Guia alimentar para crianças brasileiras menores de 2 anos/Ministério da Saúde. Secretaria de Atenção Primária à Saúde, Departamento de Promoção da Saúde. - Brasília: Ministério da Saúde, 2019.

3. Torquato HDMP, Pontes JS, Rocha CMM, et al. Amamentação na primeira hora de vida em uma maternidade de referência de Macaé. Saúde em Redes, 2019;5(1):35-47. http://dx.doi.org/10.18310/2446-4813.2019v5n1 p35-47.

4. Brasil. Ministério da Saúde. Secretaria de Atenção à Saúde. Departamento de Atenção Básica. Saúde da criança: crescimento e desenvolvimento / Ministério da Saúde. Secretaria de Atenção à Saúde. Departamento de Atenção Básica. - Brasília: Ministério da Saúde, 2012a.

5. Brasil. Ministério da Saúde. Secretaria de Política de Saúde. Organização Pan Americana da Saúde. Guia alimentar para crianças menores de dois anos/Secretaria de Políticas de Saúde, Organização Pan Americana da Saúde. Brasília: Ministério da Saúde, 2002.

6. Brasil. Ministério da Saúde. Secretaria de Atenção à Saúde. Departamento de Atenção Básica. Dez passos para uma alimentação saudável: guia alimentar para crianças menores de dois anos: um guia para o profissional da saúde na atenção básica/Ministério da Saúde, Secretaria de Atenção Básica, Departamento de Atenção Básica. - Brasília: Ministério da Saúde, 2013.

7. Boccolini CS, Boccolini PMM, Monteiro FR, et al. Tendência de indicadores do aleitamento materno no Brasil em três décadas. Rev Saúde Pública, 2017;51(108):2-9. https://doi.org/10.11606/S1518-8787.2017051000029.

8. Flores TR, Nunes BP, Neves RG, et al. Consumo de leite materno e fatores associados em crianças menores de dois anos: Pesquisa Nacional de Saúde, 2013. Cad Saude Publica, 2017;33(11):1-15. https://doi.org/10.1590/0102$311 \times 00068816$.

9. Universidade Federal do Rio De Janeiro (UFRJ). Estudo Nacional de Alimentação e Nutrição Infantil - ENANI-2019: Resultados preliminares - Indicadores de aleitamento materno no Brasil. UFRJ: Rio de Janeiro, 2020.

10. Brasil. Ministério da Saúde. Secretaria de Atenção à Saúde. Departamento de Atenção Básica. Saúde da criança: nutrição infantil: aleitamento materno e alimentação complementar/Ministério da Saúde, Secretaria de Atenção à Saúde, Departamento de Atenção Básica. - Brasília: Editora do Ministério da Saúde, 2009. 
11. Bouskelá A, Capelli JCS, Rocha CMM, LIMA FF, et al. Evolução do aleitamento materno exclusivo nos primeiros 15 anos do século XXI: um estudo no município de Macaé, Rio de Janeiro, Brasil. Demetra, 2019;14 (Supl.1):e43562. https://doi.org/10.12957/demetra.2019.43562.

12. Marinho LMF, Capelli JCS, Rocha CMM, et al. Situação da alimentação complementar de crianças entre 6 e 24 meses assistidas na rede de atenção básica de saúde de Macaé, RJ, Brasil. Cien Saude Colet, 2016;21(3):977-986. https://doi.org/10.1590/1413-81232015213.06532015.

13. Brasil. Ministério da Saúde. Dez Passos para uma Alimentação Saudável para Crianças Brasileiras Menores de dois anos. Biblioteca Virtual em Saúde do Ministério da Saúde. Brasília-DF, 2010. Disponível em: http://www.saude.gov.br/bvs. Acesso em: 13 abr. 2020.

14. Lima PG. Formação de professores: por uma ressignificação do trabalho pedagógico na escola/ Paulo Gomes Lima. - Editora EDUFGD, 2010.

15. Ribeiro ZMT, Spadella MA. Validação de conteúdo de material educativo sobre alimentação saudável para crianças menores de dois anos. Rev Paul Pediatr, 2018;36(2):155-163. https://doi.org/10.1590/1984-0462/;2018;36;2;00007.

16. Sousa CS, Turrinir NT. Validação de constructo de tecnologia educativa para pacientes mediante aplicação da técnica Delphi*. Acta Paul Enferm, 2012;25(6):990-6. https://doi.org/10.1590/S0103-21002012000600026.

17. Hoffmann T, Worrall L. Designing effective written health education materials: considerations for health professionals. Disabil Rehabil, 2004;26(19):1166-73. http://dx.doi.org/10.1080/09638280410001724816.

18. Ferraro AH, Costa EA, Vieira-da-silva LMA. A framework for health surveillance decentralization. Cad Saude Publica, 2009;25(22):1-17. https://doi.org/10.1590/s0102-311×2009001000011.

19. Souza LE, Silva LM, Hartz ZM. Conferência de consenso sobre a imagem-objetivo da descentralização da atenção à saúde no Brasil. In: Hartz ZM, Silva LM (eds.). Avaliação em saúde: dos modelos teóricos à prática na avaliação de programas e sistemas de saúde. Rio de Janeiro: Fiocruz; 2005. p. 65-102.

20. Murphy MK, Black NA, Lamping DL, et al. Consensus development methods, and their use in clinical guideline development. Health Technol Assess, 1998;2(i-iv):1-88.

21. Calderoni TL, Ribeiro YG, Sperandio N, et al. Alimentando o seu bebê - Miniguia com orientações básicas para escolhas alimentares adequadas. Macaé. Observatório da Cidade de Macaé, 1. ed., 2020.

22. Alexandre DS, Alpes MF, Reis ACMB, et al. Validação de cartilha sobre marcos do desenvolvimento da linguagem na infância. Revista CEFAC, 2020;22(2):e16219. https://doi.org/10.1590/1982-0216/202022216219.

23. Lessa LP, Silva RKSI, Rocha GA, et al. Construção de uma cartilha sobre educação no trânsito para adolescentes. Recife. Rev Enferm UFPE on line, 2018;12(10):2737-42. https://doi.org/10.5205/1981-8963-v12i10a235019p27372742-2018.

24. Almeida DM. Elaboração de materiais educativos. Disciplina Ações Educativas na Prática de Enfermagem Escola de Enfermagem da Universidade de São Paulo. São Paulo. 2017. Disponível em: https://edisciplinas.usp.br/pluginfile.php/4412041/mod_resource/content/1/ELABORA\%C3\%87\%C3\%830\%20MATE RIAL\%20EDUCATIVO.pdf. Acesso em: 22 de jan., 2020.

25. Pinto DSO, Pontes JS, Rocha CMM, Vianna MR, et al. Amamentar e alimentar na perspectiva de puérperas assistidas em uma maternidade de referência de um município do Norte Fluminense. Saúde em Redes, 2018:4(3):75-86. https://doi.org/10.18310/2446-4813.2018v4n3p75=-86.

26. Capelli JCS, Corrêa VOS, Rocha CMM, et al. Projeto IACOL: Incentivo à Alimentação Complementar Saudável aos Lactentes assistidos na Rede de Atenção Básica de Macaé. In: Lourenço AEP, Bergold LB. (Orgs.) Saberes e experiências de extensão em promoção da saúde. - Campos dos Goytacazes. Editora Essentia, 2019. p. 111-123. 
27. Capelli JCS, Rocha CMM, Braga FAMN LIMA, et al. A. Ações de promoção da alimentação saudável no primeiro ano de vida em Macaé. Rasbran, 2018;9(2):9-16.

28. Brasil. Ministério da Saúde. Secretaria de Atenção à Saúde. Departamento de Atenção Básica. Política Nacional de Atenção Básica / Ministério da Saúde. Secretaria de Atenção à Saúde. Departamento de Atenção Básica. - Brasília: Ministério da Saúde, 2012.

29. Agência Nacional de Vigilância Sanitária. ANVISA. RDC n. 259, de 20 de setembro de 2002. Disponível em: https://bvsms.saude.gov.br/bvs/saudelegis/anvisa/2002/rdc0259_20_09_2002.html. Acesso em: 22 jan. 2021.

30. Brasil. Ministério da Saúde. Secretaria de Atenção à Saúde. Área Técnica de Saúde da Criança e Aleitamento Materno. Gestões e gestores de políticas públicas de atenção à saúde da criança: 70 anos de história/Ministério da Saúde, Secretaria de Atenção à Saúde. Área Técnica de Saúde da Criança e Aleitamento Materno. - Brasília: Ministério da Saúde, 2011.

31. Cavada GS, Paiva FF, Helbig, E, Borges LR. Rotulagem nutricional: você sabe o que está comendo? Braz J Food Technol, IV SSA, 2012;84-88. https://doi.org/10.1590/S1981-67232012005000043.

32. Marins BR, Jacob SC, Peres F. Avaliação qualitativa do hábito de leitura e entendimento: recepção das informações de produtos alimentícios. Ciênc Tecnol Aliment, 2008;28(3):579-585. https://doi.org/10.1590/S010120612008000300012.

33. Brasil. Ministério da Saúde, Secretaria de Atenção à Saúde, Departamento de Atenção Básica. Guia alimentar para a população brasileira. 2a Ed. Brasília: Ministério da Saúde, 2014.

34. Macaé. Decreto n 030/2020, de 16 de março de 2020. Dispõe sobre a adoção de medidas preventivas para a contenção do coronavírus no município de Macaé. Governo do Estado do Rio de Janeiro. Prefeitura Municipal de Macaé. Disponível em: http://www.macae.rj.gov.br/midia/uploads/Decreto\%20030-2020.PDF Acesso em: 02 fev. 2021.

\section{Colaboradoras}

Calderoni TL participou da concepção do manuscrito, coleta e análise dos dados, redação e revisão crítica do manuscrito. Capelli JCS e Sperandio participaram da participou da concepção do manuscrito e análise dos dados, redação e revisão crítica do manuscrito. Ribeiro YG, Carvalho MF, Accioly E participaram redação e revisão crítica do manuscrito.

Conflito de Interesses As autoras declaram não haver conflito de interesses.

Recebido: March 17, 2021

Aceito: September 8, 2021 